\title{
Sums of Toeplitz products with harmonic symbols
}

\section{Boo Rim Choe, Hyungwoon Koo and Young Joo Lee}

\begin{abstract}
On the Bergman space of the unit disk, we consider a class of operators which contain sums of finitely many Toeplitz products with harmonic symbols. We give characterizations of when an operator in that class has finite rank or is compact. Our results provide a unified way of treating several known results.
\end{abstract}

\section{Introduction}

Let $D$ denote the unit disk of the complex plane $\mathbb{C}$. The Bergman space $L_{a}^{2}$ is the closed subspace of the usual Lebesgue space $L^{2}=L^{2}(D, A)$ consisting of all holomorphic functions on $D$ where the measure $d A$ is the normalized area measure on $D$. We let $P$ be the Hilbert space orthogonal projection from $L^{2}$ onto $L_{a}^{2}$. For a bounded measurable function $u$ on $D$, the Toeplitz operator $T_{u}$ with symbol $u$ is defined by

$$
T_{u} f=P(u f)
$$

for functions $f \in L_{a}^{2}$. Clearly, $T_{u}$ is a bounded linear operator on $L_{a}^{2}$. In this paper we are mainly concerned with harmonic symbols. So, we introduce the notation $h^{\infty}$ for the space of all bounded harmonic functions on $D$. Also, we let $H^{\infty}$ denote the space of all bounded holomorphic functions on $D$.

In a recent paper [11], Guo, Sun and Zheng characterized finite rank (semi-) commutators of two Toeplitz operators with harmonic symbols. Motivated by such results, we consider in this paper a more general class of operators which contain sums of finitely many Toeplitz products with harmonic

2000 Mathematics Subject Classification: Primary: 47B35; Secondary: 32A36.

Keywords: Toeplitz operators, Bergman space, finite rank operators. 
symbols. More explicitly, we consider operators $T$ of the form

$$
T=T_{\lambda}+\sum_{j=1}^{N} T_{u_{j}} T_{v_{j}}
$$

where $u_{j}, v_{j} \in h^{\infty}$ for each $j$ and $\lambda$ is a finite sum of finite products of $h^{\infty}$-functions. We first investigate the problem of when an operator of this type has finite rank. In addition, we also consider the problem of when such an operator is compact on $L_{a}^{2}$. Our results generalize several known results concerning (semi-) commutators of Toeplitz operators with harmonic symbols.

To state our results, we introduce some notation. Given $f, g \in L_{a}^{2}$, we let $f \otimes g$ be the rank one operator on $L_{a}^{2}$ defined by

$$
(f \otimes g) h=\langle h, g\rangle f, \quad h \in L_{a}^{2}
$$

where the notation $\langle$,$\rangle denotes the inner product in L^{2}$. We will often use the letter $z$ not only to denote points in $D$, but also to denote the identity function on $D$.

Our first result is a characterization for operators of the form (1.1) to have finite rank in terms of symbols and functions that generate their ranges. In case $\lambda=0$, our result is as follows.

Theorem 1.1. Let $u_{1}, \ldots, u_{N}, v_{1}, \ldots, v_{N} \in h^{\infty}$ and $x_{1}, \ldots, x_{n}, y_{1}, \ldots, y_{n} \in$ $L_{a}^{2}$. Then

$$
\sum_{j=1}^{N} T_{u_{j}} T_{v_{j}}=\sum_{j=1}^{n} x_{j} \otimes y_{j}
$$

if and only if the following two conditions hold:

$$
\begin{aligned}
& \text { (a) } \sum_{j=1}^{N} u_{j} v_{j}=\left(1-|z|^{2}\right)^{2} \sum_{j=1}^{n} x_{j} \overline{y_{j}} . \\
& \text { (b) } \sum_{j=1}^{N}\left[\overline{P \overline{u_{j}}}-u_{j}(0)\right]\left[P v_{j}-v_{j}(0)\right]=0 .
\end{aligned}
$$

This will be deduced as a special case of a more general result Theorem 3.5. As an immediate consequence, we give a characterization of when an operator $T$ in (1.1) is the zero operator (Theorems 3.7 and 3.8). These special cases are also new. We also apply Theorem 3.5 to recover theorems concerning finite rank sums of finitely many (semi-)commutators (Corollaries 3.9 and 3.10) and finite rank Toeplitz products (Corollary 3.11), which have been (essentially) noticed in [11]. 
Note that, although we have Theorem 1.1, whether or not there are examples of functions satisfying conditions (a) and (b) above is another separate problem in general. For example, the case $N=1$ admits only trivial examples by Corollary 3.11. When $N>1$, however, it turns out that there are nontrivial examples; see the examples at the end of Section 3.

Our next result is a characterization of compactness of operators under consideration. To state it, we introduce more notation. Given $a \in D$, we let $\varphi_{a}$ denote the standard Möbius map on $D$. Namely,

$$
\varphi_{a}(z)=\frac{a-z}{1-\bar{a} z}, \quad z \in D .
$$

Also, we let $\widetilde{\Delta}$ denote the invariant Laplacian on $D$ defined by

$$
\widetilde{\Delta} \psi=\left(1-|z|^{2}\right)^{2} \Delta \psi
$$

for $C^{2}$-functions $\psi$ on $D$ where $\Delta$ is the ordinary Laplacian. This invariant Laplacian is easily seen to be Möbius invariant by a direct calculation. The notation $C_{0}$ stands for the class of all continuous functions $\psi$ on $D$ such that $\psi(a) \rightarrow 0$ as $|a| \rightarrow 1$.

In case $\lambda=0$, our result concerning compactness is as follows.

Theorem 1.2. Let $u_{1}, \ldots, u_{N}, v_{1}, \ldots, v_{N} \in h^{\infty}$. Then the following statements are equivalent:

$$
\begin{aligned}
& \text { (a) } \sum_{j=1}^{N} T_{u_{j}} T_{v_{j}} \text { is compact. } \\
& \text { (b) } \sum_{j=1}^{N} \widetilde{\Delta}\left[\overline{P \bar{u}_{j}} P v_{j}\right] \in C_{0} \text { and } \sum_{j=1}^{N} u_{j} v_{j} \in C_{0} . \\
& \text { (c) } \sum_{j=1}^{N} u_{j} v_{j} \in C_{0} \text { and } \\
& \quad \lim _{|a| \rightarrow 1} \int_{D}\left|\sum_{j=1}^{N}\left[\overline{P\left(\overline{u_{j} \circ \varphi_{a}}\right)}-u_{j}(a)\right]\left[P\left(v_{j} \circ \varphi_{a}\right)-v_{j}(a)\right]\right| d A=0 .
\end{aligned}
$$

This will also be deduced from a more general result Theorem 4.3. As applications of Theorem 4.3, we also obtain compact versions of all the results mentioned earlier; see Theorem 4.4, Corollaries 4.5 and 4.6 . These corollaries generalize the main results in [15] and [16].

In Section 2 we collect some basic facts and known results that we use later. In Section 3 we prove a more general version of Theorem 1.1 and derive its applications. As a preliminary step, we give a characterization for harmonicity of functions of certain type. At the end of the section we construct examples related to Theorem 1.1. In Section 4 we prove compact versions of all the results obtained in Section 3. 


\section{Preliminaries}

Throughout the section we let $a \in D$ denote an arbitrary point, unless otherwise specified.

Since every point evaluation is a bounded linear functional on $L_{a}^{2}$, there corresponds to every $a \in D$ a unique function $K_{a} \in L_{a}^{2}$ which has following reproducing property:

$$
f(a)=\left\langle f, K_{a}\right\rangle
$$

for $f \in L_{a}^{2}$. The function $K_{a}$ is the well-known Bergman kernel and its explicit formula is given by

$$
K_{a}(z)=\frac{1}{(1-\bar{a} z)^{2}}, \quad z \in D .
$$

We let $k_{a}$ denote the normalized kernel, namely,

$$
k_{a}(z)=\frac{1-|a|^{2}}{(1-\bar{a} z)^{2}}, \quad z \in D
$$

By the reproducing property $(2.1)$ we see that the projection $P$ can be realized as an integral operator

$$
P u(a)=\left\langle u, K_{a}\right\rangle
$$

for $u \in L^{2}$. Moreover, this integral representation allows us to extend $P$ to $L^{1}$. It is well known that

$$
P f=f, \quad P\left(\bar{f} K_{a}\right)=\overline{f(a)} K_{a}
$$

for holomorphic functions $f \in L^{1}$. Here, $L^{p}=L^{p}(D, A)$ denotes the usual Lebesgue space. See [12, Chapter 1] for details of what have been mentioned above and related facts.

We now recall the well-known Berezin transform, which is one of the main tools in the theory of Toeplitz operators. Let $\mathfrak{L}\left(L_{a}^{2}\right)$ be the algebra of bounded linear operators on $L_{a}^{2}$. The Berezin transform of $S \in \mathfrak{L}\left(L_{a}^{2}\right)$ is the function $B[S]$ on $D$ defined by

$$
B[S](a)=\left\langle S k_{a}, k_{a}\right\rangle .
$$

For $u \in L^{\infty}$, we simply let $B u=B\left[T_{u}\right]$. Since $\left|\varphi_{a}^{\prime}(z)\right|^{2}=\left|k_{a}(z)\right|^{2}$ by a straightforward calculation, we have

$$
B u(a)=\left\langle u k_{a}, k_{a}\right\rangle=\int_{D}\left(u \circ \varphi_{a}\right) d A .
$$


The integral representation (2.3) allows us to extend the notion of the Berezin transform to functions $u \in L^{1}$. Note that the mean value property yields $B u=u$ for harmonic functions $u \in L^{1}$. Also, it is known that the Berezin transform commutes with the invariant Laplacian:

$$
B[\widetilde{\Delta} u]=\widetilde{\Delta}(B u)
$$

when $u \in L^{1} \cap C^{2}(D)$ and $\widetilde{\Delta} u \in L^{1}$; see [2, Lemma 1].

The Berezin transform turns out to provide a compactness criterion for certain classes of operators. Here, we consider operators $S$ which are finite sums of finite products of Toeplitz operators with bounded symbols. Thus, such an operator $S$ is of the form

$$
S=\sum_{i=1}^{M} T_{u_{i 1}} \cdots T_{u_{i N_{i}}}
$$

where each $u_{i j} \in L^{\infty}$. The compactness of operators of this form is characterized by the boundary vanishing property of the Berezin transform as in the next theorem.

Theorem 2.1 ([5]). Let $S$ be as in (2.5). Then $S$ is compact if and only if $B[S] \in C_{0}$.

In conjunction with Theorem 2.1 we record here the following identity for easier reference later:

$$
B\left[T_{u} T_{v}\right]-u v=B[\bar{g} h]-\bar{g} h
$$

for $u, v \in h^{\infty}$ such that $u=f+\bar{g}, v=h+\bar{k}$ where $f, g, h, k$ are holomorphic functions on $D$. This easily follows from (2.2).

Recall that the pseudohyperbolic distance between two points $z, w \in D$ is defined by $\left|\varphi_{z}(w)\right|$. Let $\mathscr{A} \subset L^{\infty}$ denote the algebra of all functions that are uniformly continuous with respect to the pseudohyperbolic distance. It is not hard to see that $h^{\infty}$-functions are Lipschitz continuous with respect to the pseudohyperbolic distance. So, in particular, we have $h^{\infty} \subset \mathscr{A}$. We remark in passing that Coburn [10] has recently proved a more general result asserting that the Berezin transform $B[S]$ of an arbitrary $S \in \mathfrak{L}\left(L_{a}^{2}\right)$ is Lipschitz continuous with respect to the pseudohyperbolic distance. For Toeplitz operators with symbols in $\mathscr{A}$, the compactness has been recently characterized by the boundary vanishing property of symbol functions as in the next theorem.

Theorem $2.2([9])$. Let $\sigma \in \mathscr{A}$. Then $T_{\sigma}$ is compact if and only if $\sigma \in C_{0}$. 
Let $U_{a}$ denote the isometry on $L_{a}^{2}$ defined by

$$
U_{a} f=\left(f \circ \varphi_{a}\right) k_{a}
$$

for $f \in L_{a}^{2}$. It is easily seen that $U_{a} U_{a}=I$ and thus $U_{a}^{-1}=U_{a}$. Now, being an invertible linear isometry, $U_{a}$ is unitary. Thus, since $k_{a}=-\varphi_{a}^{\prime}$, a direct calculation yields

$$
B\left[U_{a} S U_{a}\right]=B[S] \circ \varphi_{a}
$$

for $S \in \mathfrak{L}\left(L_{a}^{2}\right)$ and

$$
U_{a}\left(S_{1} \cdots S_{N}\right) U_{a}=\left(U_{a} S_{1} U_{a}\right) \cdots\left(U_{a} S_{N} U_{a}\right)
$$

for $S_{1}, \ldots, S_{N} \in \mathfrak{L}\left(L_{a}^{2}\right)$. Also, for $u \in L^{\infty}$, it is well known that

$$
U_{a} T_{u} U_{a}=T_{u \circ \varphi_{a}}
$$

see, for example, [4] or [5] (where $U_{a}$ is defined with an extra factor -1 ).

The following theorem is taken from [11, Theorem 2].

Theorem 2.3 ([11]). Suppose that $u \in L^{\infty}$ and $u=\sum f_{j} \overline{g_{j}}$ for finitely many holomorphic functions $f_{j}$ and $g_{j}$ on $D$. If $T_{u}$ has finite rank, then $u=0$.

\section{Finite rank operators}

In this section, we prove a more general version of Theorem 1.1, derive some applications and construct some examples. In order to do so, we give a characterization for harmonicity of functions which are finite sums of products of an holomorphic function and a co-holomorphic function. For that purpose we first make an observation that characterizes two holomorphic mappings having mutually orthogonal ranges.

We start with the well-known "complexification" lemma; see, for example, the proof of [7, Lemma 10] or the proof of [3, Theorem II].

Lemma 3.1. Let $\Omega$ be a domain in $\mathbb{C}^{n}$ and assume that $\Phi$ is holomorphic on $\Omega \times \Omega^{*}$ where $\Omega^{*}=\{\bar{z}: z \in \Omega\}$. If $\Phi(z, \bar{z})=0$ for all $z \in \Omega$, then $\Phi=0$ on $\Omega \times \Omega^{*}$.

Given a positive integer $N$, we let $I_{N}$ denote the $N \times N$ identity matrix and $S_{N}$ denote the set of all permutations on $\{1, \ldots, N\}$. Given vectors $a=\left(a_{1}, \ldots, a_{N}\right), b=\left(b_{1}, \ldots, b_{N}\right) \in \mathbb{C}^{N}$, we let $a \cdot \bar{b}=\sum_{j=1}^{N} a_{j} \overline{b_{j}}$ denote the Hermitian inner product of $a$ and $b$ on $\mathbb{C}^{N}$. Also, we let $a^{t}$ denote the 
transpose of $a$ and put $a_{\sigma}=\left(a_{\sigma_{1}}, \ldots, a_{\sigma_{N}}\right)$ for $\sigma \in S_{N}$. In the exposition below the dimension $N$ in these notations might vary and dimensions involved should be clear from the context.

Let $\Omega$ be a domain in $\mathbb{C}^{n}$ and consider holomorphic mappings

$$
F, G: \Omega \rightarrow \mathbb{C}^{N}
$$

such that the ranges are mutually orthogonal, i.e., $F \cdot \bar{G}=0$. One may easily modify the proof of $[3$, Theorem II] to see that there exists an orthonormal basis $\left\{\mu_{1}, \ldots, \mu_{N}\right\}$ of $\mathbb{C}^{N}$ such that $F$ and $G$ are of the form

$$
F=\left(F \cdot \overline{\mu_{1}}, \ldots, F \cdot \overline{\mu_{k}}, 0, \ldots, 0\right)
$$

and

$$
G=\left(0, \ldots, 0, G \cdot \overline{\mu_{k+1}}, \ldots, G \cdot \overline{\mu_{N}}\right)
$$

for some $k$ relative to the orthonormal basis $\left\{\mu_{1}, \ldots, \mu_{N}\right\}$. Here, we provide some more characterizations, which seem (to us) more concrete, as in the next theorem.

Theorem 3.2. Given finitely many holomorphic functions $f_{1}, \ldots, f_{N}$ and $g_{1}, \ldots, g_{N}$ on a domain $\Omega \subset \mathbb{C}^{n}$, put $F=\left(f_{j}\right)$ and $G=\left(g_{j}\right)$. Then the following statements are equivalent:

(a) $F \cdot \bar{G}=0$ on $\Omega$.

(b) There exist some positive integer $k<N$, some permutation $\sigma \in S_{N}$ and some $(N-k) \times k$ matrix $A$ such that

$$
F_{\sigma}^{t}=\left(\begin{array}{c}
I_{k} \\
A
\end{array}\right)\left(f_{\sigma_{1}}, \ldots, f_{\sigma_{k}}\right)^{t}
$$

and

$$
G_{\sigma}^{t}=\left(\begin{array}{c}
-A^{*} \\
I_{N-k}
\end{array}\right)\left(g_{\sigma_{k+1}}, \ldots, g_{\sigma_{N}}\right)^{t}
$$

where $A^{*}=\overline{A^{t}}$.

(c) There exist vectors $\mu_{1}, \ldots, \mu_{N}, \tau_{1}, \ldots, \tau_{N} \in \mathbb{C}^{N}$ such that

$$
F=\sum_{j=1}^{N} \mu_{j} f_{j}, \quad G=\sum_{i=1}^{N} \tau_{i} g_{i}
$$

and

$$
\mu_{i} \cdot \overline{\tau_{j}}=0, \quad i, j=1, \ldots, N
$$


Proof. We first assume (a) and prove (b). Let $\Omega^{*}=\{\bar{z}: z \in \Omega\}$ and consider a holomorphic function $\Phi$ on $\Omega \times \Omega^{*}$ defined by

$$
\Phi(z, w)=F(z) \cdot \overline{G(\bar{w})}
$$

for $(z, w) \in \Omega \times \Omega^{*}$. Then, since $\Phi(z, \bar{z})=0$ for $z \in \Omega$ by assumption, we see that $\Phi$ identically vanishes on $\Omega \times \Omega^{*}$ by Lemma 3.1.

We may assume that functions $f_{j}, g_{j}$ are all nontrivial. Choose a maximal collection of functions $\left\{f_{j_{1}}, \ldots, f_{j_{k}}\right\}$ subject to the condition that $\left\{f_{j_{1}}, \ldots, f_{j_{k}}\right\}$ is linearly independent. Note that $\left\{f_{1}, \ldots, f_{N}\right\}$ is linearly dependent, because $\Phi=0$. So, we have $k<N$. Put $m=N-k$ for convenience. Now, after permutation if necessary, we may assume that $\left\{f_{1}, \ldots, f_{k}\right\}$ is linearly independent. Now, since $\left\{f_{1}, \ldots, f_{k}, f_{j}\right\}$ is linearly dependent for each $j>k$ by maximality, there exists some $m \times k$ matrix $A$ such that

$$
\left(f_{k+1}, \ldots, f_{N}\right)^{t}=A\left(f_{1}, \ldots, f_{k}\right)^{t}
$$

which yields the desired representation of $F$. Put $\widetilde{F}=\left(f_{1}, \ldots, f_{k}\right)$. Then, inserting the above into the identity $\Phi=0$, we have

$$
0=F(z) \cdot \overline{G(w)}=\widetilde{F}(z)\left(I_{k}, A^{t}\right) \overline{G(w)^{t}}
$$

for $z, w \in \Omega$. Since $\left\{f_{1}, \ldots, f_{k}\right\}$ is linearly independent, it follows that

$$
\left(I_{k}, A^{t}\right) \overline{G^{t}}=0
$$

or equivalently,

$$
\left(g_{1}, \ldots, g_{k}\right)^{t}=-A^{*}\left(g_{k+1}, \ldots, g_{N}\right)^{t}
$$

which yields the desired representation of $G$.

Next, we assume (b) and prove (c). Put $m=N-k$. Let $a_{i}=$ $\left(a_{i 1}, \ldots, a_{i k}\right)$ be the $i$-th row of $A$ and $b_{j}=\left(a_{1 j}, \ldots, a_{m j}\right)^{t}$ be the $j$-th column of $A$. Also, let $e_{\ell}^{j}$ be the $j$-th row of $I_{\ell}$. Then we have by assumption

$$
F_{\sigma}=\sum_{j=1}^{k}\left(e_{k}^{j}, b_{j}^{t}\right) f_{\sigma_{j}} \quad \text { and } \quad G_{\sigma}=\sum_{i=1}^{m}\left(-\overline{a_{i}}, e_{m}^{i}\right) g_{\sigma_{k+i}} .
$$

So, taking vectors

$$
\widetilde{\mu}_{j}= \begin{cases}\left(e_{k}^{j}, b_{j}^{t}\right) & \text { if } j \leq k \\ 0 & \text { if } j>k\end{cases}
$$

and

$$
\widetilde{\tau}_{i}= \begin{cases}0 & \text { if } i \leq k \\ \left(-\overline{a_{i}}, e_{m}^{i}\right) & \text { if } i>k\end{cases}
$$


we obtain

$$
F_{\sigma}=\sum_{j=1}^{N} \widetilde{\mu}_{j} f_{\sigma_{j}} \quad \text { and } \quad G_{\sigma}=\sum_{i=1}^{N} \widetilde{\tau}_{i} g_{\sigma_{i}}
$$

Note that

$$
\widetilde{\mu}_{j} \cdot \overline{\widetilde{\tau}_{i}}=\left(e_{k}^{j}\right) \cdot \overline{\left(-\overline{a_{i}}\right)}+\left(b_{j}^{t}\right) \cdot \overline{\left(e_{m}^{i}\right)}=-a_{i j}+a_{i j}=0
$$

for all $i>k$ and $j \leq k$. So, (c) holds.

Finally, the implication (c) $\Longrightarrow$ (a) is straightforward. The proof is complete.

Now, we give the following characterization, which will be a key tool in proving Theorem 1.1.

Theorem 3.3. Let $f_{1}, \ldots, f_{N}$ and $g_{1}, \ldots, g_{N}$ be finitely many holomorphic functions on $D$. If $\sum_{j=1}^{N} f_{j} \overline{g_{j}}$ is harmonic on $D$, then

$$
\sum_{j=1}^{N}\left(f_{j}-f_{j}(0)\right)\left(\overline{g_{j}}-\overline{g_{j}(0)}\right)=0
$$

holds on $D$.

Proof. Let $F=\left(f_{j}\right)$ and $G=\left(g_{j}\right)$. Assuming $F(0)=G(0)=0$ without loss of generality, we need to prove $F \cdot \bar{G}=0$ on $D$. Since $\sum_{j=1}^{N} f_{j} \overline{g_{j}}$ is harmonic on $D$, we have $F^{\prime} \cdot \overline{G^{\prime}}=0$ on $D$. It follows from Theorem 3.2 that there exist vectors $\mu_{1}, \ldots, \mu_{N}, \tau_{1}, \ldots, \tau_{N} \in \mathbb{C}^{N}$ such that

$$
F^{\prime}=\sum_{j=1}^{N} \mu_{j} f_{j}^{\prime}, \quad G^{\prime}=\sum_{i=1}^{N} \tau_{i} g_{i}^{\prime}
$$

and

$$
\mu_{i} \cdot \overline{\tau_{j}}=0, \quad i, j=1, \ldots, N .
$$

Now, since $F(0)=G(0)=0$, we have

$$
F=\sum_{j=1}^{N} \mu_{j} f_{j}, \quad G=\sum_{i=1}^{N} \tau_{i} g_{i}
$$

and thus

$$
F \cdot \bar{G}=\sum_{i, j=1}^{N}\left(\mu_{i} \cdot \overline{\tau_{j}}\right) f_{i} \overline{g_{j}}=0
$$

as desired. The proof is complete. 
The Bloch space $\mathcal{B}$ is the space of all holomorphic functions $f$ on $D$ for which

$$
\sup _{z \in D}\left(1-|z|^{2}\right)\left|f^{\prime}(z)\right|<\infty .
$$

It is easily seen that Bloch functions are of logarithmic growth near the boundary and thus $\mathcal{B} \subset L^{p}$ for all $0<p<\infty$. In particular, we have

$$
f \bar{g} \in L^{2} \quad \text { and } \quad \widetilde{\Delta}(f \bar{g}) \in L^{\infty}
$$

for functions $f, g \in \mathcal{B}$. Also, it is well known that, given a function $u=$ $f+\bar{g} \in h^{\infty}$ where $f, g$ are holomorphic functions on $D$, we have $f, g \in \mathcal{B}$; see, for example, [15].

In what follows, given $u, v \in h^{\infty}$, we let

$$
Q_{u, v}(\cdot, a)=\left[\overline{P\left(\overline{u \circ \varphi_{a}}\right)}-u(a)\right]\left[P\left(v \circ \varphi_{a}\right)-v(a)\right]
$$

for $a \in D$ and put

$$
R_{u, v}=Q_{v, u}
$$

More explicitly, if $u=f+\bar{g}$ and $v=h+\bar{k}$ where $f, g, h, k \in \mathcal{B}$, then

$$
\begin{aligned}
& Q_{u, v}(\cdot, a)=\left[\overline{g \circ \varphi_{a}}-\overline{g(a)}\right]\left[h \circ \varphi_{a}-h(a)\right] \\
& R_{u, v}(\cdot, a)=\left[f \circ \varphi_{a}-f(a)\right]\left[\overline{k \circ \varphi_{a}}-\overline{k(a)}\right]
\end{aligned}
$$

for $a \in D$. Also, we let $\mathscr{F}$ denote the class of all functions $\lambda$ of the form

$$
\lambda=\sum_{i=1}^{M} u_{i_{1}} u_{i_{2}} \cdots u_{i_{N_{i}}}
$$

where each $u_{i_{j}} \in h^{\infty}$. We need the following simple fact.

Lemma 3.4. $\widetilde{\Delta} \lambda \in L^{\infty}$ for each $\lambda \in \mathscr{F}$.

Proof. Let $\lambda \in \mathscr{F}$. We may assume $\lambda=u_{1} \cdots u_{N}$ where each $u_{j} \in h^{\infty}$. Let $\partial=\frac{\partial}{\partial z}$ and put

$$
\begin{aligned}
\|\lambda\|_{*} & =\sup _{z \in D}\left(1-|z|^{2}\right)[|\partial \lambda(z)|+|\bar{\partial} \lambda(z)|], \\
\|\lambda\|_{* *} & =\sup _{z \in D}\left(1-|z|^{2}\right)^{2}|\partial \bar{\partial} \lambda(z)|
\end{aligned}
$$

for simplicity. It is clear that $\|\lambda\|_{*}<\infty$ and $\|\lambda\|_{* *}<\infty$ in case $N=1$. Now, given $u \in h^{\infty}$, an elementary calculation yields inequalities

$$
\begin{aligned}
\|\lambda u\|_{*} & \leq\|u\|_{\infty}\|\lambda\|_{*}+\|\lambda\|_{\infty}\|u\|_{*}, \\
\|\lambda u\|_{* *} & \leq\|u\|_{\infty}\|\lambda\|_{* *}+\|\lambda\|_{*}\|u\|_{*} .
\end{aligned}
$$

So, an induction on $N$ shows that $\|\lambda\|_{*}<\infty$ and thus $\|\lambda\|_{* *}<\infty$ for arbitrary $N$. The proof is complete. 
Now, we are ready to prove the following more general version of Theorem 1.1.

Theorem 3.5. Let $u_{1}, \ldots, u_{N}, v_{1}, \ldots, v_{N} \in h^{\infty}, x_{1}, \ldots, x_{n}, y_{1}, \ldots, y_{n} \in L_{a}^{2}$ and $\lambda \in \mathscr{F}$. Then

$$
T_{\lambda}+\sum_{j=1}^{N} T_{u_{j}} T_{v_{j}}=\sum_{j=1}^{n} x_{j} \otimes y_{j}
$$

if and only if the following two conditions hold:

(a) $\lambda+\sum_{j=1}^{N} u_{j} v_{j}=\left(1-|z|^{2}\right)^{2} \sum_{j=1}^{n} x_{j} \overline{y_{j}}$.

(b) $\lambda+\sum_{j=1}^{N} \overline{P \overline{u_{j}}} P v_{j}$ is harmonic.

The idea of the proof of the necessity below comes from the argument in $[11]$.

Proof. For each $j=1, \ldots, N$, we write $u_{j}=f_{j}+\overline{g_{j}}$ and $v_{j}=h_{j}+\overline{k_{j}}$ where $f_{j}, g_{j}, h_{j}$ and $k_{j}$ are all in $\mathcal{B}$. Also, we write

$$
\left(1-|z|^{2}\right)^{2} \sum_{j=1}^{n} x_{j} \bar{y}_{j}=\left(1-2 z \bar{z}+z^{2} \bar{z}^{2}\right) \sum_{j=1}^{n} x_{j} \bar{y}_{j}=\sum_{j=1}^{3 n} \alpha_{j} \overline{\beta_{j}}
$$

where $\alpha_{j}, \beta_{j}$ are all in $L_{a}^{2}$.

First suppose (3.4) holds. Note that we have by (2.6)

$$
B\left[T_{u_{j}} T_{v_{j}}\right]=B\left[h_{j} \overline{g_{j}}\right]+\left(f_{j} h_{j}+\overline{g_{j}} \overline{k_{j}}\right)+f_{j} \overline{k_{j}}
$$

for each $j$. Also, note that

$$
B\left[x_{j} \otimes y_{j}\right]=\left(1-|z|^{2}\right)^{2} x_{j} \overline{y_{j}}
$$

for each $j$. Thus, taking the Berezin transforms of both sides of (3.4), we obtain

$$
B \lambda+\sum_{j=1}^{N} B\left[h_{j} \overline{g_{j}}\right]+\sum_{j=1}^{N}\left(f_{j} h_{j}+\overline{g_{j}} \overline{k_{j}}\right)+\sum_{j=1}^{N} f_{j} \overline{k_{j}}=\sum_{j=1}^{3 n} \alpha_{j} \overline{\beta_{j}} .
$$

Let

$$
\sigma=\widetilde{\Delta} \lambda+\sum_{j=1}^{N} \widetilde{\Delta}\left(h_{j} \overline{g_{j}}\right)
$$


Note that $\sigma$ is bounded by (3.1) and Lemma 3.4. Now, applying the invariant Laplacian to both sides of (3.5), we have by (2.4)

$$
B \sigma=\sum_{j=1}^{3 n} \widetilde{\Delta}\left(\alpha_{j} \overline{\beta_{j}}\right)-\sum_{j=1}^{N} \widetilde{\Delta}\left(f_{j} \overline{k_{j}}\right)=\left(1-|z|^{2}\right)^{2}\left(\sum_{j=1}^{3 n} \alpha_{j}^{\prime} \overline{\beta_{j}^{\prime}}-\sum_{j=1}^{N} f_{j}^{\prime} \overline{k_{j}^{\prime}}\right)
$$

Dividing by $\left(1-|z|^{2}\right)^{2}$, we obtain

$$
\int_{D} \frac{\sigma(\zeta)}{|1-z \bar{\zeta}|^{4}} d A(\zeta)=\sum_{j=1}^{3 n} \alpha_{j}^{\prime}(z) \overline{\beta_{j}^{\prime}(z)}-\sum_{j=1}^{N} f_{j}^{\prime}(z) \overline{k_{j}^{\prime}(z)}
$$

for $z \in D$. Now, by Lemma 3.1, we have

$$
\int_{D} \frac{\sigma(\zeta)}{(1-z \bar{\zeta})^{2}(1-\bar{w} \zeta)^{2}} d A(\zeta)=\sum_{j=1}^{3 n} \alpha_{j}^{\prime}(z) \overline{\beta_{j}^{\prime}(w)}-\sum_{j=1}^{N} f_{j}^{\prime}(z) \overline{k_{j}^{\prime}(w)}
$$

for every $z, w \in D$. Differentiate both sides of the above as many times as needed with respect to $\bar{w}$ variable and then insert $w=0$. The result is

$$
\begin{aligned}
T_{\sigma} \zeta^{\ell}(z) & =\int_{D} \frac{\sigma(\zeta) \zeta^{\ell}}{(1-z \bar{\zeta})^{2}} d A(\zeta) \\
& =\sum_{j=1}^{3 n} a_{j \ell} \alpha_{j}^{\prime}(z)+\sum_{j=1}^{N} b_{j \ell} f_{j}^{\prime}(z), \quad \ell=0,1,2, \ldots
\end{aligned}
$$

for some coefficients $a_{j \ell}$ and $b_{j \ell}$. Now, by the same argument as in the proof of [11, Proposition 4], we see that $T_{\sigma}$ has finite rank. Note that $\sigma$ can be represented as a sum of finitely many products of a holomorphic function and a co-holomorphic function, because $\lambda \in \mathscr{F}$. So, Theorem 2.3 gives $\sigma=0$. Namely, the function $\lambda+\sum_{j=1}^{N} h_{j} \overline{g_{j}}$ is harmonic. Accordingly, noting that

$$
P v_{j}=h_{j}+\overline{k_{j}(0)} \text { and } P \overline{u_{j}}=g_{j}+\overline{f_{j}(0)},
$$

we conclude (b). Also, it follows from (3.6) that the function

$$
\sum_{j=1}^{N} f_{j} \overline{k_{j}}-\sum_{j=1}^{3 n} \alpha_{j} \overline{\beta_{j}}
$$

is harmonic. Since harmonic $L^{1}$-functions are invariant under the Berezin transform, it follows that

$$
\sum_{j=1}^{N} f_{j} \overline{k_{j}}-\sum_{j=1}^{3 n} \alpha_{j} \overline{\beta_{j}}=\sum_{j=1}^{N} B\left[f_{j} \overline{k_{j}}\right]-\sum_{j=1}^{3 n} B\left[\alpha_{j} \overline{\beta_{j}}\right] .
$$


Combining this with (3.5), we obtain

$$
\begin{aligned}
B \lambda+\sum_{j=1}^{N} B\left[u_{j} v_{j}\right] & =B \lambda+\sum_{j=1}^{N}\left(f_{j} h_{j}+\overline{g_{j} k_{j}}\right)+\sum_{j=1}^{N} B\left[f_{j} \overline{k_{j}}+h_{j} \overline{g_{j}}\right] \\
& =\sum_{j=1}^{N} B\left[f_{j} \overline{k_{j}}\right]+\sum_{j=1}^{3 n} \alpha_{j} \overline{\beta_{j}}-\sum_{j=1}^{N} f_{j} \overline{k_{j}} \\
& =\sum_{j=1}^{3 n} B\left[\alpha_{j} \overline{\beta_{j}}\right] .
\end{aligned}
$$

So, we conclude (a), because the Berezin transform is one-to-one (see, for example, [12, Chapter 2]).

Now, suppose (a) and (b). Note that the set $\left\{K_{a}: a \in D\right\}$ spans a dense subset of $L_{a}^{2}$. So, to prove (3.4), it is sufficient to show

$$
\left[T_{\lambda}+\sum_{j=1}^{N} T_{u_{j}} T_{v_{j}}\right] K_{a}=\sum_{j=1}^{n}\left(x_{j} \otimes y_{j}\right) K_{a}
$$

for all $a \in D$. Let $a \in D$ be an arbitrary point. First, note that we have by $(2.2)$

$$
\begin{aligned}
T_{u_{j}} T_{v_{j}} K_{a} & =P\left[\left(f_{j}+\overline{g_{j}}\right)\left(h_{j}+\overline{k_{j}(a)}\right) K_{a}\right] \\
& =P\left[\left(f_{j} h_{j}+h_{j} \overline{g_{j}}+\overline{g_{j}(a) k_{j}(a)}\right) K_{a}\right]+f_{j} \overline{k_{j}(a)} K_{a} \\
& =P\left[\left(f_{j} h_{j}+h_{j} \overline{g_{j}}+\overline{g_{j} k_{j}}\right) K_{a}\right]+f_{j} \overline{k_{j}(a)} K_{a} \\
& =P\left[\left(u_{j} v_{j}-f_{j} \overline{k_{j}}\right) K_{a}\right]+f_{j} \overline{k_{j}(a)} K_{a}
\end{aligned}
$$

for each $j$. Also, note that $\left(x_{j} \otimes y_{j}\right) K_{a}=x_{j} \overline{y_{j}(a)}$ for each $j$. So, by (a), in order to prove (3.8), it is necessary and sufficient to show

$$
\sum_{j=1}^{3 n} P\left[\alpha_{j} \overline{\beta_{j}} K_{a}\right]-\sum_{j=1}^{N} P\left[f_{j} \overline{k_{j}} K_{a}\right]=\sum_{j=1}^{n} x_{j} \overline{y_{j}(a)}-\sum_{j=1}^{N} f_{j} \overline{k_{j}(a)} K_{a} .
$$

Since the function $\lambda+\sum_{j=1}^{N} h_{j} \overline{g_{j}}$ is harmonic by (b) and (3.7), the function $\lambda+\sum_{j=1}^{N}\left(u_{j} v_{j}-f_{j} \overline{k_{j}}\right)$ is also harmonic. Note that $\sum_{j=1}^{N}\left(u_{j} v_{j}-f_{j} \overline{k_{j}}\right) \in L^{2}$, because $f_{j} k_{j} \in L_{a}^{2}$ by (3.1) for each $j$. Thus, we have by (a)

$$
\sum_{j=1}^{3 n} \alpha_{j} \overline{\beta_{j}}-\sum_{j=1}^{N} f_{j} \overline{k_{j}}=F+\bar{G}
$$

for some holomorphic functions $F, G \in L_{a}^{2}$. 
Thus, multiplying by $K_{a}$ and then applying the projection $P$ to both sides of the above, we obtain by $(2.2)$

$$
\sum_{j=1}^{3 n} P\left[\alpha_{j} \overline{\beta_{j}} K_{a}\right]-\sum_{j=1}^{N} P\left[f_{j} \overline{k_{j}} K_{a}\right]=[F+\overline{G(a)}] K_{a}
$$

Meanwhile, we have by Lemma 3.1

$$
F+\overline{G(a)}=\sum_{j=1}^{3 n} \alpha_{j} \overline{\beta_{j}(a)}-\sum_{j=1}^{N} f_{j} \overline{k_{j}(a)}=K_{a}^{-1} \sum_{j=1}^{n} x_{j} \overline{y_{j}(a)}-\sum_{j=1}^{N} f_{j} \overline{k_{j}(a)}
$$

Combining these equalities, we obtain (3.9). The proof is complete.

Taking $\lambda=0$ in Theorem 3.5, we obtain Theorem 1.1 which we restate here for convenience.

Theorem 3.6. Let $u_{1}, \ldots, u_{N}, v_{1}, \ldots, v_{N} \in h^{\infty}$ and $x_{1}, \ldots, x_{n}, y_{1}, \ldots, y_{n} \in L_{a}^{2}$. Then

$$
\sum_{j=1}^{N} T_{u_{j}} T_{v_{j}}=\sum_{j=1}^{n} x_{j} \otimes y_{j}
$$

if and only if the following two conditions hold:
(a) $\sum_{j=1}^{N} u_{j} v_{j}=\left(1-|z|^{2}\right)^{2} \sum_{j=1}^{n} x_{j} \overline{y_{j}}$.
(b) $\sum_{j=1}^{N} Q_{u_{j}, v_{j}}(\cdot, 0)=0$.

Proof. The theorem follows from Theorem 3.5 and the fact that

$$
\sum_{j=1}^{N} \overline{P \overline{u_{j}}} P v_{j}
$$

is harmonic if and only if (b) holds by Theorem 3.3.

As another special case of Theorem 3.5, we have the following characterizations for operators under consideration to be the zero operator.

Theorem 3.7. Let $u_{1}, \cdots, u_{N}, v_{1}, \cdots, v_{N} \in h^{\infty}$ and $\lambda \in \mathscr{F}$. Then

$$
T_{\lambda}+\sum_{j=1}^{N} T_{u_{j}} T_{v_{j}}=0
$$

if and only if the following two conditions hold: 
(a) $\lambda+\sum_{j=1}^{N} u_{j} v_{j}=0$.

(b) $\sum_{j=1}^{N} R_{u_{j}, v_{j}}(\cdot, 0)=0$.

Proof. If $\lambda+\sum_{j=1}^{N} u_{j} v_{j}=0$, then it is easily seen that $\lambda+\sum_{j=1}^{N} \overline{P \overline{u_{j}}} P v_{j}$ is harmonic if and only if $\sum_{j=1}^{N} P u_{j} \overline{P \overline{v_{j}}}$ is harmonic. Thus the theorem holds by Theorems 3.5 and 3.3. The proof is complete.

In case $N=1$ Theorem 3.7 is known to hold for general $\lambda \in L^{\infty}$ (see [1, Corollary 1]) and we do not know whether such a general result holds for arbitrary $N$.

Combining Theorems 3.6 and 3.7, we have the following characterization.

Theorem 3.8. Let $u_{1}, \ldots, u_{N}, v_{1}, \ldots, v_{N} \in h^{\infty}$. Then the following statements are equivalent:
(a) $\sum_{j=1}^{N} T_{u_{j}} T_{v_{j}}=0$.
(b) $\sum_{j=1}^{N} T_{v_{j}} T_{u_{j}}=0$.
(c) $\sum_{j=1}^{N} Q_{u_{j}, v_{j}}(\cdot, 0)=\sum_{j=1}^{N} u_{j} v_{j}=0$.
(d) $\sum_{j=1}^{N} R_{u_{j}, v_{j}}(\cdot, 0)=\sum_{j=1}^{N} u_{j} v_{j}=0$.

We now apply our theorems to recover results in [11] concerning sums of finitely many (semi-)commutators. Given Toeplitz operators $T_{u}$ and $T_{v}$, we let

$$
\begin{aligned}
& {\left[T_{u}, T_{v}\right]=T_{u} T_{v}-T_{v} T_{u},} \\
& \left(T_{u}, T_{v}\right]=T_{u} T_{v}-T_{u v}
\end{aligned}
$$

denote the commutator and the semi-commutator, respectively.

Theorem 3.5 also has some consequences for sums of finitely many (semi-) commutators of Toeplitz operators with harmonic symbols as in the next two corollaries. For semi-commutators, we have the following consequence, which is a slightly different form of [11, Theorem 8]. 
Corollary 3.9. Let $u_{1}, \ldots, u_{N}, v_{1}, \ldots, v_{N} \in h^{\infty}$. Then the following statements are equivalent:
(a) $\sum_{\substack{j=1 \\ N}}^{N}\left(T_{u_{j}}, T_{v_{j}}\right]=0$.
(b) $\sum_{j=1}^{N}\left(T_{u_{j}}, T_{v_{j}}\right]$ has finite rank.
(c) $\sum_{j=1}^{N} R_{u_{j}, v_{j}}(\cdot, 0)=0$.

Proof. The equivalence (a) $\Longleftrightarrow$ (c) holds by Theorem 3.7 (with $\lambda=$ $-\sum_{j=1}^{N} u_{j} v_{j}$ ). The implication (a) $\Longrightarrow(\mathrm{b})$ is trivial.

We now assume (b) and prove (a). Since $\sum_{j=1}^{N}\left(T_{u_{j}}, T_{v_{j}}\right]$ has finite rank, we have $\sum_{j=1}^{N}\left(T_{u_{j}}, T_{v_{j}}\right]=\sum_{j=1}^{n} x_{j} \otimes y_{j}$ for some functions $x_{1}, \ldots, x_{n}, y_{1}, \ldots, y_{n} \in$ $L_{a}^{2}$. We may assume that $x_{1}, \ldots, x_{n}$ are linearly independent. We have $\sum_{j=1}^{n} x_{j} \overline{y_{j}}=0$ by Theorem 3.5 and thus

$$
\sum_{j=1}^{n} x_{j}(z) \overline{y_{j}(w)}=0
$$

for all $z, w \in D$ by Lemma 3.1. Since $x_{1}, \ldots, x_{n}$ are linearly independent, it follows that $y_{j}=0$ for all $j$ and thus $\sum_{j=1}^{N}\left(T_{u_{j}}, T_{v_{j}}\right]=0$. The proof is complete.

Since a commutator is the difference of associated semi-commutators, Corollary 3.9 yields yet another corollary for commutators as follows. Another way of deriving this corollary is to take $\lambda=0$ in Theorem 3.5 (or Theorem 3.7).

Corollary 3.10. Let $u_{1}, \ldots, u_{N}, v_{1}, \ldots, v_{N} \in h^{\infty}$. Then the following statements are equivalent:
(a) $\sum_{j=1}^{N}\left[T_{u_{j}}, T_{v_{j}}\right]=0$.
(b) $\sum_{j=1}^{N}\left[T_{u_{j}}, T_{v_{j}}\right]$ has finite rank.
(c) $\sum_{j=1}^{N} Q_{u_{j}, v_{j}}(\cdot, 0)=\sum_{j=1}^{N} R_{u_{j}, v_{j}}(\cdot, 0)$.

Also, we can recover the result on finite rank Toeplitz products with harmonic symbols, which is proved in [11, Theorem 7].

Corollary 3.11. Let $u, v \in h^{\infty}$. Then $T_{u} T_{v}$ has finite rank if and only if either $u=0$ or $v=0$. 
Proof. The sufficiency is trivial. We prove the necessity. Suppose that $T_{u} T_{v}$ has finite rank. Let $u=f+\bar{g}$ and $v=h+\bar{k}$ where $f, g, h, k$ are holomorphic functions. By Theorem 3.6 we have

(i) $u v=\left(1-|z|^{2}\right)^{2} \sum_{j=1}^{n} x_{j} \overline{y_{j}}$

(ii) $h \bar{g}$ is harmonic on $\bar{D}$

for some finitely many functions $x_{1}, \ldots, x_{n}, y_{1}, \ldots, y_{n} \in L_{a}^{2}$. It follows from (i) that $u v$ has a continuous extension on $\bar{D}$ and $u v=0$ on $\partial D$ (see $[14$, Theorem 7.2.5]). Being bounded harmonic functions, $u$ and $v$ have radial limits almost everywhere on $\partial D$. So, there are two possibilities: one is that $u$ or $v$ vanishes almost everywhere on $\partial D$ and the other is that $u$ and $v$ vanish on some sets of positive measures on $\partial D$. Note that $u$ or $\bar{v}$ is holomorphic by (ii). Therefore, in either case, we conclude either $u=0$ or $v=0$ on $D$. The proof is complete.

In view of Theorem 3.6, one may ask whether (3.10) can actually happen. In other words, one may ask whether there are examples of functions satisfying conditions (a) and (b) of Theorem 3.6. The answer is yes. For example, given $x_{1}, \ldots, x_{n}, y_{1}, \ldots, y_{n} \in H^{\infty}$, put

$$
\begin{aligned}
& u_{j 1}=x_{j}, \quad u_{j 2}=-2 z x_{j}, \quad u_{j 3}=z^{2} x_{j} \\
& v_{j 1}=\overline{y_{j}}, \quad v_{j 2}=\bar{z} \overline{y_{j}}, \quad v_{j 3}=\bar{z}^{2} \overline{y_{j}}
\end{aligned}
$$

for $j=1, \ldots, n$. Then one can easily check that conditions (a) and (b) of Theorem 3.6 are satisfied and thus

$$
\sum_{j=1}^{n} \sum_{i=1}^{3} T_{u_{j i}} T_{v_{j i}}=\sum_{j=1}^{n} x_{j} \otimes y_{j} .
$$

In particular, the operator

$$
I-2 T_{z} T_{\bar{z}}+T_{z^{2}} T_{\bar{z}^{2}}=1 \otimes 1
$$

is simply the point evaluation at the origin, which one may also verify by a direct calculation.

The above examples shows that, given an $n$-dimensional subspace $X_{n}$ of $L_{a}^{2}$ generated by bounded holomorphic functions, we can find $3 n$ pairs of symbols $u_{j}, v_{j} \in h^{\infty}$ such that the range of $\sum_{j=1}^{3 n} T_{u_{j}} T_{v_{j}}$ is precisely $X_{n}$. In prescribing ranges like that, we do not know whether we can control the number of pairs of symbols in general. However, as far as the rank is concerned, the next example shows that just two pairs of symbols are enough to produce arbitrary ranks. Note that at least two pairs of symbols are required in prescribing ranks by Corollary 3.11.

Example 3.12. Given a positive integer $n$, there exist some $u_{1}, u_{2}, v_{1}, v_{2} \in$ $h^{\infty}$ such that $\sum_{j=1}^{2} T_{u_{j}} T_{v_{j}}$ has rank $n$. 
Proof. Let a positive integer $n$ be given and let $p_{n}$ be the polynomial of degree $(n-1)$ such that

$$
z^{n+1}-(n+1) z+n=(z-1)^{2} p_{n} .
$$

An elementary calculation yields

$$
p_{n}=\sum_{j=0}^{n-1}\left(z^{j}+z^{j-1}+\cdots+1\right)=\sum_{j=0}^{n-1}(n-j) z^{j} .
$$

Choose real numbers $a, b$ such that $|a|+|b|<n$ and put

$$
y=\frac{1}{a z^{n+1}+b z+n} .
$$

Note that $y \in H^{\infty}$, because $|a|+|b|<n$. We may choose $a, b$ with the additional property that the polynomials $z^{n+1}-a$ and $(n+1) z+b$ have no common zeros and therefore we have

$$
\left|z^{n+1}-a\right|+|(n+1) z+b| \geq \delta, \quad z \in D
$$

for some positive number $\delta$. So, there exist some functions $h_{1}, h_{2} \in H^{\infty}$ such that

$$
\left(z^{n+1}-a\right) h_{1}-((n+1) z+b) h_{2}=1
$$

on $D$ by the corona theorem.

Now, given a nontrivial function $x \in H^{\infty}$, take functions $f_{j}, k_{j} \in H^{\infty}$ as follows:

$$
\begin{aligned}
& f_{1}=\left(z^{n+1}-a\right) x, \quad f_{2}=-((n+1) z+b) x \\
& k_{1}=z^{n+1} y, \quad k_{2}=z y .
\end{aligned}
$$

Using these functions, we put

$$
u_{j}=f_{j}, \quad v_{j}=h_{j}+\overline{k_{j}}, \quad j=1,2 .
$$

Then we have by $(3.7)$

$$
\overline{P \overline{u_{j}}}-u_{j}(0)=\overline{f_{j}(0)}-\overline{f_{j}(0)}=0
$$

for $j=1,2$ and

$$
\begin{aligned}
u_{1} v_{1}+u_{2} v_{2} & =\left(f_{1} h_{1}+f_{2} h_{2}\right)+f_{1} \overline{k_{1}}+f_{2} \overline{k_{2}} \\
& =x+\left(z^{n+1}-a\right) \bar{z}^{n+1} x \bar{y}-((n+1) z+b) \bar{z} x \bar{y} \\
& =x \bar{y}\left[\bar{y}^{-1}+\left(z^{n+1}-a\right) \bar{z}^{n+1}-((n+1) z+b) \bar{z}\right] \\
& =\left(1-|z|^{2}\right)^{2} p_{n}\left(|z|^{2}\right) x \bar{y} .
\end{aligned}
$$


Thus, setting

$$
x_{j}=(n-j) z^{j} x, \quad y_{j}=z^{j}
$$

for $j=0, \ldots, n-1$, we obtain

$$
u_{1} v_{1}+u_{2} v_{2}=\left(1-|z|^{2}\right)^{2} \sum_{j=0}^{n-1} x_{j} \bar{y}_{j}
$$

on $D$. Now, having (3.13) and (3.14), we conclude

$$
T_{u_{1}} T_{v_{1}}+T_{u_{2}} T_{v_{2}}=\sum_{j=0}^{n-1} x_{j} \otimes y_{j}
$$

by Theorem 3.6. Since $\left\{x_{j}\right\}$ and $\left\{y_{j}\right\}$ are both linearly independent, this shows that the operator $\sum_{j=1}^{2} T_{u_{j}} T_{v_{j}}$ has rank $n$. The proof is complete.

\section{Compact operators}

In this section, we prove compact versions of results obtained in the previous section. For that purpose, we first recall the notion of maximal ideal space. The maximal ideal space $\mathfrak{M}$ of $H^{\infty}$ is the space (endowed with the weak* topology of the dual of $H^{\infty}$ ) of all nonzero multiplicative linear functionals on $H^{\infty}$. As is well known, we have $H^{\infty} \subset C(\mathfrak{M})$ via the Gelfand transform. Moreover, it is known $\left(\left[13\right.\right.$, Lemma 4.4]) that $h^{\infty} \subset C(\mathfrak{M})$. We will use the same notation for a function $u \in h^{\infty}$ and its continuous extension $u$ on the whole $\mathfrak{M}$. Identifying $z \in D$ with the multiplicative evaluation functional $f \mapsto f(z)$, we can freely regard $D$ as a subset of $\mathfrak{M}$. The corona theorem says that $D$ is dense in $\mathfrak{M}$.

For each $m \in \mathfrak{M}, \mathrm{K}$. Hoffman $([13])$ constructed a canonical map $L_{m}$ from $D$ into $\mathfrak{M}$. This map $L_{m}$ is defined by taking a net $\left\{z_{\alpha}\right\}$ in $D$ such that $z_{\alpha} \rightarrow m$ and defining

$$
L_{m}(z)(h)=\lim _{\alpha} h \circ \varphi_{z_{\alpha}}(z)
$$

for $z \in D$ and $h \in H^{\infty}$. The above limit exists and is independent of the net $\left\{z_{\alpha}\right\}$ provided that $z_{\alpha} \rightarrow m$. For each $f \in H^{\infty}$, the map $f \circ L_{m}$ is in $H^{\infty}$. Moreover, if $u$ is continuous on $\mathfrak{M}$ and $\left\{z_{\alpha}\right\}$ is a net converging to $m$ in $\mathfrak{M}$, then it is known ([15, Lemma 5]) that $u \circ \varphi_{z_{\alpha}} \rightarrow u \circ L_{m}$ uniformly on compact subsets of $D$ and thus

$$
(\widetilde{\Delta} u) \circ \varphi_{z_{\alpha}}=\widetilde{\Delta}\left(u \circ \varphi_{z_{\alpha}}\right) \rightarrow \widetilde{\Delta}\left(u \circ L_{m}\right) .
$$

In particular, we have $u \circ L_{m} \in h^{\infty}$ for $u \in h^{\infty}$. Also, $\lambda \circ L_{m} \in \mathscr{F}$ for $\lambda \in \mathscr{F}$; recall that $\mathscr{F}$ is the class introduced in (3.3). 
The following lemma is implicit in the proof of [6, Lemma 5.1].

Lemma 4.1. Suppose that $\left\{z_{\alpha}\right\}$ is a net in $D$ such that $z_{\alpha} \rightarrow m \in \mathfrak{M}$. Then

$$
\widetilde{\Delta}[\overline{P \bar{u}} P v] \circ \varphi_{z_{\alpha}} \rightarrow \widetilde{\Delta}\left[\overline{P\left(\overline{u \circ L_{m}}\right)} P\left(v \circ L_{m}\right)\right]
$$

(pointwise) on $D$ for $u, v \in h^{\infty}$.

Also, we need the following fact.

Lemma 4.2. Suppose that $\left\{z_{\alpha}\right\}$ is a net in $D$ such that $z_{\alpha} \rightarrow m \in \mathfrak{M}$. Let $\lambda_{1}, \ldots, \lambda_{M} \in \mathscr{F}$. Then

$$
T_{\lambda_{M} \circ \varphi_{w_{\alpha}}} \cdots T_{\lambda_{1} \circ \varphi_{w_{\alpha}}} \rightarrow T_{\lambda_{M} \circ L_{m}} \cdots T_{\lambda_{1} \circ L_{m}}
$$

in the weak operator topology.

Proof. Fix $f \in L_{a}^{2}$. Recall $\lambda_{1} \circ \varphi_{w_{\alpha}} \rightarrow \lambda_{1} \circ L_{m}$ uniformly on compact subsets of $D$. So, since $\lambda_{1}$ is bounded, the dominated convergence theorem yields

$$
\left(\lambda_{1} \circ \varphi_{w_{\alpha}}\right) f \rightarrow\left(\lambda_{1} \circ L_{m}\right) f \quad \text { in } \quad L^{2}
$$

and thus

$$
P\left[\left(\lambda_{1} \circ \varphi_{w_{\alpha}}\right) f\right] \rightarrow P\left[\left(\lambda_{1} \circ L_{m}\right) f\right] \quad \text { in } \quad L_{a}^{2}
$$

by continuity of $P$. This proves the lemma for $M=1$. We now proceed by induction on $M$. Assume $M \geq 2$ and suppose that the lemma holds for $M-1$. Put

$$
h_{\alpha}=T_{\lambda_{M-1} \circ \varphi_{w_{\alpha}}} \cdots T_{\lambda_{1} \circ \varphi_{w_{\alpha}}} f \quad \text { and } \quad g=T_{\lambda_{M-1} \circ L_{m}} \cdots T_{\lambda_{1} \circ L_{m}} f
$$

for simplicity. Then we have by induction hypothesis $h_{\alpha} \rightarrow g$ in $L_{a}^{2}$ and thus uniformly on compact subsets of $D$. Now, since $\lambda_{M} \circ \varphi_{w_{\alpha}}$ is bounded and converges pointwise to $\lambda_{M} \circ L_{m}$, we have

$$
\begin{aligned}
\left\|\left(\lambda_{M} \circ \varphi_{w_{\alpha}}\right) h_{\alpha}-\left(\lambda_{M} \circ L_{m}\right) g\right\|_{L^{2}} \\
\quad \leq\left\|\lambda_{M}\right\|_{L^{\infty}}\left\|h_{\alpha}-g\right\|_{L^{2}}+\left\|\left(\lambda_{M} \circ \varphi_{w_{\alpha}}\right) g-\left(\lambda_{M} \circ L_{m}\right) g\right\|_{L^{2}} \rightarrow 0
\end{aligned}
$$

by the dominated convergence theorem and thus

$$
P\left[\left(\lambda_{M} \circ \varphi_{w_{\alpha}}\right) h_{\alpha}\right] \rightarrow P\left[\left(\lambda_{M} \circ L_{m}\right) g\right] \quad \text { in } \quad L_{a}^{2}
$$

by continuity of $P$. In other words, $T_{\lambda_{M} \circ \varphi_{w_{\alpha}}} h_{\alpha} \rightarrow T_{\lambda_{M} \circ L_{m}} g$ in $L_{a}^{2}$. This completes the induction and the proof of the lemma.

We are now ready to prove the compact version of Theorem 3.7. 
Theorem 4.3. Let $u_{1}, \ldots, u_{N}, v_{1}, \ldots, v_{N} \in h^{\infty}$ and $\lambda \in \mathscr{F}$. Then the following statements are equivalent:

(a) $T_{\lambda}+\sum_{j=1}^{N} T_{u_{j}} T_{v_{j}}$ is compact.

(b) $T_{\lambda_{\circ} L_{m}}+\sum_{j=1}^{N} T_{u_{j} \circ L_{m}} T_{v_{j} \circ L_{m}}=0$ for each $m \in \mathfrak{M} \backslash D$.

(c) $\widetilde{\Delta} \lambda+\sum_{j=1}^{N} \widetilde{\Delta}\left[\overline{P \bar{u}_{j}} P v_{j}\right] \in C_{0}$ and $\lambda+\sum_{j=1}^{N} u_{j} v_{j} \in C_{0}$.

(d) $\sum_{j=1}^{N} \widetilde{\Delta}\left[P u_{j} \overline{P \bar{v}_{j}}\right] \in C_{0}$ and $\lambda+\sum_{j=1}^{N} u_{j} v_{j} \in C_{0}$.

(e) $\sum_{j=1}^{N}\left(T_{u_{j}}, T_{v_{j}}\right]$ is compact and $\lambda+\sum_{j=1}^{N} u_{j} v_{j} \in C_{0}$.

(f) $\lim _{|a| \rightarrow 1} \int_{D}\left|\sum_{j=1}^{N} R_{u_{j}, v_{j}}(z, a)\right| d A(z)=0$ and $\lambda+\sum_{j=1}^{N} u_{j} v_{j} \in C_{0}$.

We will complete the proof by proving the following sequences of implications:

$$
\begin{aligned}
& (\mathrm{b}) \Longleftrightarrow(\mathrm{c}), \\
& (\mathrm{b}) \Longleftrightarrow(\mathrm{d}), \\
& (\mathrm{a}) \Longrightarrow(\mathrm{b}) \Longrightarrow(\mathrm{e}) \Longrightarrow(\mathrm{a}), \\
& (\mathrm{b}) \Longrightarrow(\mathrm{f}) \Longrightarrow(\mathrm{e}) .
\end{aligned}
$$

Since proofs are somewhat long, we will prove each case separately.

Proof of (b) $\Longleftrightarrow(\mathbf{c})$. First we assume (b) and prove (c). It is sufficient to show that, for a given net $\left\{w_{\alpha}\right\}$ in $D$ converging to some $m \in \mathfrak{M} \backslash D$, we have

$$
\widetilde{\Delta}\left[\lambda+\sum_{j=1}^{N} \overline{P \overline{u_{j}}} P v_{j}\right]\left(w_{\alpha}\right) \rightarrow 0
$$

and

$$
\left[\lambda+\sum_{j=1}^{N} u_{j} v_{j}\right]\left(w_{\alpha}\right) \rightarrow 0 .
$$

So, fix a net $\left\{w_{\alpha}\right\}$ in $D$ such that $w_{\alpha} \rightarrow m$ for some $m \in \mathfrak{M} \backslash D$. To prove (4.2), note that we have

$$
\widetilde{\Delta}\left[\lambda \circ L_{m}+\sum_{j=1}^{N} \overline{P\left(\overline{u_{j} \circ L_{m}}\right)} P\left(v_{j} \circ L_{m}\right)\right]=0
$$


by assumption (b) and Theorem 3.5. Thus, we have (4.2) by (4.1) and Lemma 4.1 (with evaluation at the origin). Also, note that

$$
\left[\lambda+\sum_{j=1}^{N} u_{j} v_{j}\right] \circ L_{m}=0
$$

holds by assumption (b) and Theorem 3.5. Thus, we have (4.3) by a similar argument.

Now, we assume (c) and prove (b). Let $m \in \mathfrak{M} \backslash D$ and choose a net $\left\{w_{\alpha}\right\}$ in $D$ such that $w_{\alpha} \rightarrow m$. Fix an arbitrary point $a \in D$. Put $z_{\alpha}=\varphi_{w_{\alpha}}(a)$ and $m_{a}=L_{m}(a)$. Since $h\left(z_{\alpha}\right)=h\left(\varphi_{w_{\alpha}}(a)\right) \rightarrow m_{a}(h)$ for $h \in H^{\infty}$, we have $z_{\alpha} \rightarrow m_{a}$ in $\mathfrak{M}$.

By the Schwarz lemma there are rotations, say $W_{a, \alpha}$, such that

$$
\varphi_{z_{\alpha}}=\varphi_{w_{\alpha}} \circ \varphi_{a} \circ W_{a, \alpha} .
$$

Since the set of rotations is compact, we may assume $W_{a, \alpha}$ converges to some rotation $W_{a}$. Now, for a given function $f \in H^{\infty}$, since $f \circ \varphi_{w_{\alpha}} \rightarrow f \circ L_{m}$ uniformly on compact subsets of $D$, we see that $f \circ \varphi_{z_{\alpha}} \rightarrow f \circ L_{m} \circ \varphi_{a} \circ W_{a}$ on $D$. Thus, $L_{m_{a}}=L_{m} \circ \varphi_{a} \circ W_{a}$. It follows that

$$
\widetilde{\Delta}\left[\overline{P\left(\overline{u \circ L_{m_{a}}}\right)} P\left(v \circ L_{m_{a}}\right)\right]=\widetilde{\Delta}\left[\overline{P\left(\overline{u \circ L_{m}}\right)} P\left(v \circ L_{m}\right)\right] \circ \varphi_{a} \circ W_{a}
$$

for $u, v \in h^{\infty}$ by the Möbius invariance of $\widetilde{\Delta}$.

Note that $m \in \mathfrak{M} \backslash D$ implies $\left|w_{\alpha}\right| \rightarrow 1$ and thus $\left|z_{\alpha}\right| \rightarrow 1$. Now, since $\left|z_{\alpha}\right| \rightarrow 1$, we obtain by (4.1), (4.4) and Lemma 4.1 (with evaluation at the origin)

$0=\lim _{\alpha} \widetilde{\Delta}\left[\lambda+\sum_{j=1}^{N} \overline{P \bar{u}_{j}} P v_{j}\right]\left(z_{\alpha}\right)=\widetilde{\Delta}\left[\lambda \circ L_{m}+\sum_{j=1}^{N} \overline{P\left(\overline{u_{j} \circ L_{m}}\right)} P\left(v_{j} \circ L_{m}\right)\right](a)$.

Since $a \in D$ is arbitrary, this shows that $\lambda \circ L_{m}+\sum_{j=1}^{N} \overline{P\left(\overline{u_{j} \circ L_{m}}\right)} P\left(v_{j} \circ L_{m}\right)$ is harmonic. Also, since $\lambda+\sum_{j=1}^{N} u_{j} v_{j} \in C_{0}$, we have $\lambda \circ L_{m}+\sum_{j=1}^{N}\left(u_{j} v_{j}\right) \circ$ $L_{m}=0$. Hence, by Theorem 3.5, we conclude (b). The proof is complete.

Proof of (b) $\Longleftrightarrow(\mathbf{d})$. By Theorems 3.7 and 3.3 we have (b) if and only if

$$
\left[\lambda+\sum_{j=1}^{N} u_{j} v_{j}\right] \circ L_{m}=0
$$

and

$$
\widetilde{\Delta}\left[\sum_{j=1}^{N} P\left(u_{j} \circ L_{m}\right) \overline{P\left(\overline{v_{j} \circ L_{m}}\right)}\right]=0
$$

for each $m \in \mathfrak{M} \backslash D$. Thus, following the proof of (b) $\Longleftrightarrow(\mathrm{c})$, we see that (b) and (d) are equivalent. The proof is complete. 
Proof of $(\mathbf{a}) \Longrightarrow(\mathbf{b}) \Longrightarrow(\mathbf{e}) \Longrightarrow(\mathbf{a})$. First, we assume (a) and prove (b). Let $m \in \mathfrak{M} \backslash D$. Since the set $\left\{k_{a}: a \in D\right\}$ spans a dense subset of $L_{a}^{2}$, it is sufficient to show that

$$
\left[T_{\lambda \circ L_{m}}+\sum_{j=1}^{N} T_{u_{j} \circ L_{m}} T_{v_{j} \circ L_{m}}\right] k_{a}=0, \quad a \in D .
$$

Fix $a \in D$ and choose a net $\left\{w_{\alpha}\right\}$ in $D$ such that $w_{\alpha} \rightarrow m$. Then, since

$$
T_{\lambda \circ \varphi_{w_{\alpha}}}+\sum_{j=1}^{N} T_{u_{j} \circ \varphi_{w_{\alpha}}} T_{v_{j} \circ \varphi_{w_{\alpha}}} \longrightarrow T_{\lambda \circ L_{m}}+\sum_{j=1}^{N} T_{u_{j} \circ L_{m}} T_{v_{j} \circ L_{m}}
$$

in the weak operator topology by Lemma 4.2, we have

$$
\begin{aligned}
& \left\|\left[T_{\lambda \circ L_{m}}+\sum_{j=1}^{N} T_{u_{j} \circ L_{m}} T_{v_{j} \circ L_{m}}\right] k_{a}\right\|_{L^{2}}=\lim _{\alpha}\left\|\left[T_{\lambda \circ \varphi_{w_{\alpha}}}+\sum_{j=1}^{N} T_{u_{j} \circ \varphi_{w_{\alpha}}} T_{v_{j} \circ \varphi_{w_{\alpha}}}\right] k_{a}\right\|_{L^{2}} \\
& \quad=\lim _{\alpha}\left\|U_{w_{\alpha}}\left[T_{\lambda}+\sum_{j=1}^{N} T_{u_{j}} T_{v_{j}}\right] U_{w_{\alpha}} k_{a}\right\|_{L^{2}} \quad \text { (by (2.8) and (2.9)) } \\
& \quad=\lim _{\alpha}\left\|\left[T_{\lambda}+\sum_{j=1}^{N} T_{u_{j}} T_{v_{j}}\right] U_{w_{\alpha}} k_{a}\right\|_{L^{2}} .
\end{aligned}
$$

Note that $\left|w_{\alpha}\right| \rightarrow 1$, because $m \in \mathfrak{M} \backslash D$. Thus, it is easily seen that $U_{w_{\alpha}} k_{a}$ converges to 0 weakly in $L_{a}^{2}$. Hence, the compactness of $T_{\lambda}+\sum_{j=1}^{N} T_{u_{j}} T_{v_{j}}$ yields (4.5).

Next, we assume (b) and prove (e). The second part of assertion (e) is contained in the implication $(\mathrm{b}) \Longrightarrow(\mathrm{c})$, which is proved above. By Theorem 2.2 we see that $T_{\lambda}+T_{\sum_{j=1}^{N} u_{j} v_{j}}$ is compact. So, in order to prove (e), we need to prove that $T_{\lambda}+\sum_{j=1}^{N} T_{u_{j}} T_{v_{j}}$ is compact. By Theorem 2.1, it is sufficient to prove

$$
B\left[T_{\lambda}+\sum_{j=1}^{N} T_{u_{j}} T_{v_{j}}\right] \in C_{0}
$$

Suppose not. Then there is a net $\left\{w_{\alpha}\right\}$ in $D$ converging to some $m \in \mathfrak{M} \backslash D$ such that

$$
\underset{\alpha}{\limsup }\left|B\left[T_{\lambda}+\sum_{j=1}^{N} T_{u_{j}} T_{v_{j}}\right]\left(w_{\alpha}\right)\right|>0
$$


Note that we have by Lemma 4.2

$$
B\left[T_{\lambda \circ \varphi_{w_{\alpha}}}+\sum_{j=1}^{N} T_{u_{j} \circ \varphi_{w_{\alpha}}} T_{v_{j} \circ \varphi_{w_{\alpha}}}\right] \longrightarrow B\left[T_{\lambda \circ L_{m}}+\sum_{j=1}^{N} T_{u_{j} \circ L_{m}} T_{v_{j} \circ L_{m}}\right]
$$

pointwise on $D$. It follows that

$$
\begin{aligned}
0 & =B\left[T_{\lambda \circ L_{m}}+\sum_{j=1}^{N} T_{u_{j} \circ L_{m}} T_{v_{j} \circ L_{m}}\right](0) \\
& =\lim _{\alpha} B\left[T_{\lambda \circ \varphi_{w_{\alpha}}}+\sum_{j=1}^{N} T_{u_{j} \circ \varphi_{w_{\alpha}}} T_{v_{j} \circ \varphi_{w_{\alpha}}}\right](0) \\
& =\lim _{\alpha} B\left[U_{w_{\alpha}}\left(T_{\lambda}+\sum_{j=1}^{N} T_{u_{j}} T_{v_{j}}\right) U_{w_{\alpha}}\right](0) \\
& =\lim _{\alpha} B\left[T_{\lambda}+\sum_{j=1}^{N} T_{u_{j}} T_{v_{j}}\right]\left(\varphi_{w_{\alpha}}(0)\right) \\
& =\lim _{\alpha} B\left[T_{\lambda}+\sum_{j=1}^{N} T_{u_{j}} T_{v_{j}}\right]\left(w_{\alpha}\right)
\end{aligned}
$$

which contradicts (4.7). Hence we have (4.6), as desired.

Finally, the implication (e) $\Longrightarrow$ (a) holds by Theorem 2.2 . The proof is complete.

Before proceeding, we recall the well-known notion of Hankel operators. For $u \in L^{\infty}$, the Hankel operator $H_{u}$ with symbol $u$ is the operator on $L_{a}^{2}$ defined by $H_{u} f=(I-P)(u f)$. The relation between Toeplitz operators and Hankel operators is given by the well-known identity: $\left(T_{u}, T_{v}\right]=H_{\bar{u}}^{*} H_{v}$. Using this identity, one can easily verify that the semi-commutator of Toeplitz operators $T_{u}$ and $T_{v}$ with harmonic symbols are represented as an integral operator:

$$
\left(T_{u}, T_{v}\right] f(a)=\int_{D} \frac{\Lambda_{u, v}(z, a)}{(1-a \bar{z})^{2}} f(z) d A(z), \quad a \in D
$$

where

$$
\Lambda_{u, v}(z, a)=[P u(z)-P u(a)][\overline{P \bar{v}(z)}-\overline{P \bar{v}(a)}] .
$$

The kernel $\Lambda_{u, v}$ is closely related with $R_{u, v}$ in the sense that

$$
\Lambda_{u, v}\left(\varphi_{a}(z), a\right)=R_{u, v}(z, a),
$$

which can be seen by a little manipulation. 
Proof of $(\mathbf{b}) \Longrightarrow(\mathbf{f}) \Longrightarrow(\mathbf{e})$. First, we assume (b) and prove (f). We only need to prove the first part of (f). Suppose that the first part of (f) fails to hold. Then there is a net $\left\{w_{\alpha}\right\}$ in $D$ converging to some $m \in \mathfrak{M} \backslash D$ such that

$$
\underset{\alpha}{\lim \sup _{0}} \int_{D}\left|\sum_{j=1}^{N} R_{u_{j}, v_{j}}\left(z, w_{\alpha}\right)\right| d A(z)>0 .
$$

Note that we have by Lemma 4.2

$$
P\left(u \circ \varphi_{w_{\alpha}}\right)-u\left(w_{\alpha}\right) \longrightarrow P\left(u \circ L_{m}\right)-u \circ L_{m}(0) \quad \text { in } \quad L_{a}^{2}
$$

for each $u \in h^{\infty}$. Thus, applying this to functions $u_{j}$ and $\overline{v_{j}}$, we obtain

$$
\begin{aligned}
& \sum_{j=1}^{N} R_{u_{j}, v_{j}}\left(\cdot, w_{\alpha}\right) \longrightarrow \\
& \quad \sum_{j=1}^{N}\left[P\left(u_{j} \circ L_{m}\right)-u_{j} \circ L_{m}(0)\right]\left[\overline{P\left(\overline{v_{j} \circ L_{m}}\right)}-v_{j} \circ L_{m}(0)\right]
\end{aligned}
$$

in $L^{1}$. Meanwhile, we have by (b) and Theorem 3.7

$$
\sum_{j=1}^{N}\left[P\left(u_{j} \circ L_{m}\right)-u_{j} \circ L_{m}(0)\right]\left[\overline{P\left(\overline{v_{j} \circ L_{m}}\right)}-v_{j} \circ L_{m}(0)\right]=0,
$$

which, together with (4.9), is a contradiction to (4.8).

Next, we assume (f) and prove (e). For each $r \in(0,1)$, define $S_{r}: L_{a}^{2} \rightarrow$ $L^{2}$ by

$$
S_{r} f(a)=\chi_{r D}(a) \int_{D} \frac{\Lambda(z, a)}{(1-a \bar{z})^{2}} f(z) d A(z), \quad a \in D
$$

for $f \in L_{a}^{2}$ where $\Lambda=\sum_{j=1}^{N} \Lambda_{u_{j}, v_{j}}$ and $\chi_{r D}$ denotes the usual characteristic function of the set $r D$. Now, following the proof of of [8, Theorem 1] (or, easily modifying the proof of [6, Theorem 1.3]), one can verify that each $S_{r}$ is compact and that

$$
\left\|\sum_{j=1}^{N}\left(T_{u_{j}}, T_{v_{j}}\right]-S_{r}\right\|^{2} \leq C \sup _{a \in D \backslash r D}\left\{\int_{D}\left|\Lambda\left(\varphi_{a}(z), a\right)\right| d A(z)\right\}^{1 / 14}
$$

for some constant $C$ independent of $r$.

Note that $\Lambda\left(\varphi_{a}(z), a\right)=\sum_{j=1}^{N} R_{u_{j}, v_{j}}(z, a)$. So, the operator $\sum_{j=1}^{N}\left(T_{u_{j}}, T_{v_{j}}\right]$ is approximated in the strong operator topology by compact operators, so it is compact. The proof is complete. 
In case $N=1$ some characterizations in Theorem 4.3 are already known for more general $\lambda$. Namely, the conditions (a), (d) and (e) are known to be equivalent for general $\lambda \in \mathscr{A}$, and a version for general $\lambda \in L^{\infty}$ is also known; see [9, Theorem 4.4]. We do not know whether such general results hold for arbitrary $N$.

In case $\lambda=0$ in Theorem 4.3, note that we have

$$
\sum_{j=1}^{N} T_{u_{j} \circ L_{m}} T_{v_{j} \circ L_{m}}=0 \Longleftrightarrow \sum_{j=1}^{N} T_{v_{j} \circ L_{m}} T_{u_{j} \circ L_{m}}=0
$$

for $m \in \mathfrak{M} \backslash D$ by Theorem 3.8. Thus we have the following consequence of Theorem 4.3, which contains Theorem 1.2 and is the compact version of Theorem 3.8.

Theorem 4.4. Let $u_{1}, \ldots, u_{N}, v_{1}, \ldots, v_{N} \in h^{\infty}$. Then the following statements are equivalent:
(a) $\sum_{j=1}^{N} T_{u_{j}} T_{v_{j}}$ is compact.
(b) $\sum_{j=1}^{N} \widetilde{\Delta}\left[\overline{P \bar{u}_{j}} P v_{j}\right] \in C_{0}$ and $\sum_{j=1}^{N} u_{j} v_{j} \in C_{0}$.
(c) $\lim _{|a| \rightarrow 1} \int_{D}\left|\sum_{j=1}^{N} Q_{u_{j}, v_{j}}(z, a)\right| d A(z)=0$ and $\sum_{j=1}^{N} u_{j} v_{j} \in C_{0}$.

Moreover, each of the above conditions is equivalent to the similar condition with $u_{j}$ and $v_{j}$ replaced by each other.

Remark. One may also directly prove the implication (c) $\Longrightarrow$ (a) in the above theorem as follows. Note that we have by (3.2), (2.3) and (2.6)

$$
\sum_{j=1}^{N} \int_{D} Q_{u_{j}, v_{j}}(z, a) d A(z)=\sum_{j=1}^{N}\left(B\left[T_{u_{j}} T_{v_{j}}\right]-u_{j} v_{j}\right)(a)
$$

for $a \in D$. So, assuming (c), we have $\sum_{j=1}^{N}\left(B\left[T_{u_{j}} T_{v_{j}}\right]-u_{j} v_{j}\right) \in C_{0}$. Combining this with the assumption $\sum_{j=1}^{N} u_{j} v_{j} \in C_{0}$, we have $\sum_{j=1}^{N} B\left[T_{u_{j}} T_{v_{j}}\right] \in C_{0}$. So, (a) holds by Theorem 2.1.

Another special case $\lambda=-\sum_{j=1}^{N} u_{j} v_{j}$ in Theorem 4.3 yields the following corollary for sums of finitely many semi-commutators, which is the compact version of Corollary 3.9. 
Corollary 4.5. Let $u_{1}, \ldots, u_{N}, v_{1}, \ldots, v_{N} \in h^{\infty}$. Then the following statements are equivalent:

(a) $\sum_{j=1}^{N}\left(T_{u_{j}}, T_{v_{j}}\right]$ is compact.

(b) $\sum_{j=1}^{N} \widetilde{\Delta}\left[P u_{j} \overline{P \overline{v_{j}}}\right] \in C_{0}$.

(c) $\lim _{|a| \rightarrow 1} \int_{D}\left|\sum_{j=1}^{N} R_{u_{j}, v_{j}}(z, a)\right| d A(z)=0$.

For sums of finitely many commutators, one can use Theorem 4.4 or Corollary 4.5 to derive the following compact version of Corollary 3.10.

Corollary 4.6. Let $u_{1}, \ldots, u_{N}, v_{1}, \ldots, v_{N} \in h^{\infty}$. Then the following statements are equivalent:
(a) $\sum_{j=1}^{N}\left[T_{u_{j}}, T_{v_{j}}\right]$ is compact.
(b) $\sum_{j=1}^{N} \widetilde{\Delta}\left[\overline{P \overline{u_{j}}} P v_{j}-P u_{j} \overline{P \overline{v_{j}}}\right] \in C_{0}$.
(c) $\lim _{|a| \rightarrow 1} \int_{D}\left|\sum_{j=1}^{N} Q_{u_{j}, v_{j}}(z, a)-R_{u_{j}, v_{j}}(z, a)\right| d A(z)=0$.

\section{References}

[1] Ahern, P.: On the range of the Berezin transform. J. Funct. Anal. 215 (2004), no. 1, 206-216.

[2] Ahern, P. And ČuČKović, Z̆.: A theorem of Brown-Halmos type for Bergman space Toeplitz operators. J. Funct. Anal. 187 (2001), no. 1, 200210.

[3] Ahern, P. and Rudin, W.: M-harmonic products. Indag. Math. (N.S.) 2 (1991), no. 2, 141-147.

[4] Axler, S. AND C̆UČKOvić, Z̆.: Commuting Toeplitz operators with harmonic symbols. Integral Equations Operator Theory 14 (1991), 1-11.

[5] Axler, S. And Zheng, D.: Compact operators via the Berezin transform. Indiana Univ. Math. J. 47 (1998), no. 2, 387-400.

[6] Choe, B. R., Koo, H. And LeE, Y. J.: Commuting Toeplitz operators on the polydisk. Trans. Amer. Math. Soc. 356 (2004), no. 5, 1727-1749. 
[7] Choe, B. R. And Lee, Y.J.: Pluriharmonic symbols of commuting Toeplitz operators. Illinois J. Math. 37 (1993), no. 3, 424-436.

[8] Choe, B. R. And Lee, Y. J.: Pluriharmonic symbols of essentially commuting Toeplitz operators. Illinois J. Math. 42 (1998), no. 2, 280-293.

[9] Choe, B., Lee, Y. J., Nam, K. And Zheng, D.: Products of Bergman space Toeplitz operators on the polydisk. Math. Ann. 337 (2007), 295-316.

[10] Coburn, L.: A Lipschitz estimate for Berezin's operator calculus. Proc. Amer. Math. Soc. 133 (2005), no. 1, 127-131.

[11] Guo, K., Sun, S. And Zheng, D.: Finite rank commutators and semicommutators of Toeplitz operators with harmonic symbols. Illinois J. Math. 51 (2007), no. 2, 583-596.

[12] Hedenmalm, H., Korenblum, B. and Zhu, K.: Theory of Bergman space. Graduate Text in Math. 199. Springer-Verlag, New York, 2000.

[13] Hoffman, K.: Bounded analytic functions and Gleason parts. Ann. of Math. (2) 86 (1967), 74-111.

[14] Rudin, W.: Function theory in the unit ball of $\mathbb{C}^{n}$. Grundlehren der Mathematischen Wissenschaften 241. Springer-Verlag, New York-Berlin, 1980.

[15] Stroethoff, K.: Essentially commuting Toeplitz operators with harmonic symbols. Can. J. Math. 45 (1993), no. 5, 1080-1093.

[16] Zheng, D.: Hankel operators and Toeplitz operators on the Bergman space. J. Funct. Anal. 83 (1989), no. 1, 98-120.

Recibido: 21 de diciembre de 2005

$$
\begin{array}{r}
\text { Boo Rim Choe } \\
\text { Department of Mathematics } \\
\text { Korea University } \\
\text { Seoul 136-713, Korea } \\
\text { cbr@korea.ac.kr } \\
\text { Hyungwoon Koo } \\
\text { Department of Mathematics } \\
\text { Korea University } \\
\text { Seoul 136-713, Korea } \\
\text { koohw@korea. ac.kr } \\
\text { Young Joo Lee } \\
\text { Department of Mathematics } \\
\text { Chonnam National University } \\
\text { Gwangju 500-757, Korea } \\
\text { leeyj@chonnam.ac.kr }
\end{array}
$$

This research was supported by KOSEF(R01-2003-000-10243-0). 